\title{
A physics-based fitting and extrapolation method for measured impact ionization coefficients in III-V semiconductors
}

\author{
Hin-Fai Chau and Dimitris Pavlidis \\ Center for High-Frequency Microelectronics, Solid-State Electronics Laboratory, Department of Electrical \\ Engineering and Computer Science, The University of Michigan, Ann Arbor, Michigan 48104-2122
}

(Received 18 October 1991; accepted for publication 31 March 1992)

\begin{abstract}
A general approach based on a physical model of impact ionization to fit and extrapolate measured ionization coefficients of electrons $\alpha$ and holes $\beta$ in III-V semiconductors is described. Materials being considered include $\mathrm{GaAs}, \mathrm{Al}_{x} \mathrm{Ga}_{1-x} \mathrm{As}(x=0.1-0.4)$, InP, $\mathrm{In}_{0.53} \mathrm{Ga}_{0.47} \mathrm{As}$, and $\mathrm{In}_{0.52} \mathrm{Al}_{0.48} \mathrm{As}$. Expressions giving the correct dependencies are obtained at very large or small electric fields outside the range of most measurements while at the same time a reasonable fit is achieved for experimental data. The results of the proposed approach yielded a set of physical parameters, which can be coupled with the temiperature-dependence relationships in the model to predict impact ionization coefficients over a wide range of electric fields at different temperatures, and can be useful in calculations of temperature-dependent avalanche breakdown voltages of electronic and optical devices.
\end{abstract}

\section{INTRODUCTION}

Numerous reports have been published in the past on measurements of the impact ionization coefficients of electrons and holes in different III-V semiconductors. The selection of the most reliable dependencies is, however, often a difficult task. ${ }^{1}$ Since most experimental investigations have been carried out in a very narrow range of electric fields, extrapolation has to be used in low and high fields not considered in the measurements, but often required for simulating the performance of various microwave or optical devices. The impact ionization coefficients of electrons $\alpha$ and holes $\beta$ are in most cases expressed empirically as

$$
\alpha, \beta=A \exp \left[-(B / F)^{m}\right]
$$

where $A, B$, and $m$ are selected for best agreement with experimental measurements of the ionization rate, and $F$ is the electric field. This equation holds only over a limited range of $F$ because of the simplistic model on which it is based. Extrapolation of $\alpha$ and $\beta$ in electric-field ranges not considered in the measurements often gives incorrect tendency at high or low fields. ${ }^{2}$ This limits the usefulness of this approach in analyzing, for example, the breakdown characteristics of a device.

For materials and electric-field ranges where $\alpha$ and $\beta$ have not been experimentally determined, it is necessary to make use of a theoretical model in order to evaluate the field-dependent characteristics of the ionization coeffcients. Section II brieffy reviews various theoretical models available for impact ionization. The approach adopted in this paper is based on Okuto and Crowell's theoretical model. ${ }^{3}$ Analytic models regarding the estimation of impact ionization threshold energy are also presented. The deficiencies of the simple empirical formula [Eq. (1)] are described in Sec. III. Section IV presents results based on the developed approach as applied to published data on the measured impact ionization coefficients in III-V semicon- ductors, such as GaAs, $\mathrm{Al}_{x} \mathrm{Ga}_{1-x} \mathrm{As}(x=0.1-0.4)$, InP, $\mathrm{In}_{0.53} \mathrm{Ga}_{0.47} \mathrm{As}$, and $\mathrm{In}_{0.52} \mathrm{Al}_{0.48} \mathrm{As}$.

\section{THEORETICAL MODELS}

A generalized theory regarding the impact ionization process in semiconductors has been developed and discussed by Baraff. ${ }^{4}$ It is based on the numerical solution of the time-dependent Boltzmann transport equation and makes use of a general formula for $\alpha$ and $\beta$ in terms of three physical parameters, namely, the ionization threshold energy $E_{i}$, the optical phonon energy or average energy loss per phonon scattered $E_{n}$ and the mean free path for optical phonon scattering $\lambda$. The results of this theory agree well with both Shockley's low-field results ${ }^{5}$ and Wolff's high-field limit ${ }^{6}$ of the impact ionization process.

While Baraff's model has been widely used in avalanche studies, it tends to give incorrect results at very large electric fields, as discussed by Okuto and Crowell. ${ }^{3}$ To overcome these problems, Okuto and Crowell developed a simple analytic expression for $\alpha$ and $\beta$ that is valid over a wide range of electric fields: ${ }^{3}$

$$
\begin{aligned}
\alpha, \beta= & \left(\frac{q F}{E_{i}}\right) \exp \left(0.217\left(\frac{E_{i}}{E_{r}}\right)^{1.14}-\left\{\left[0.217\left(\frac{E_{i}}{E_{r}}\right)^{1.14}\right]^{2}\right.\right. \\
& \left.\left.+\left(\frac{E_{i}}{q F \lambda}\right)^{2}\right\}^{1 / 2}\right),
\end{aligned}
$$

where $E_{i}, E_{n}$ and $\lambda$ are defined as above and $F$ is the electric field. In practice, $E_{r}$ and $\lambda$ may have a slight dependence on electric field. ${ }^{7}$ As the electric field dependence of $E_{r}$ and $\lambda$ is small in comparison to the uncertainties of the measured ionization coefficients, these parameters were assumed to be constant.

The temperature dependence of $\alpha$ and $\beta$ can be found with the help of the following equations: ${ }^{8}$ 


$$
\begin{aligned}
& E_{r}=E_{r 0} \tanh \left(E_{r 0} / 2 k T\right), \\
& \lambda=\lambda_{0} \tanh \left(E_{r 0} / 2 k T\right),
\end{aligned}
$$

where $E_{r 0}$ and $\lambda_{0}$ arc $E_{r}$ and $\lambda$ at $0 \mathrm{~K}$. Furthermore, the temperature dependence of $E_{i}$ can be assumed to be the same as the temperature dependence of the band-gap energy $E_{g}{ }^{3,9}$

In principle, one is able to completely determine the avalanche breakdown characteristics of a material once $E_{i}$, $E_{n}$ and $\lambda$ are known. Numerous models have been proposed to estimate the impact ionization threshold energy $E_{i}$ in various materials. Depending on the band structure, different approximations have been used. Closed-form expressions can be obtained only for simple parabolic band ap- proximations, ${ }^{10-12}$ and they are summarized in the Appendix for both electron- and hole-initiated mechanisms, as well as for direct and indirect band-gap materials. However, for the actual band structures evaluated from pseudopotential calculations, the threshold energy $E_{i}$ has to be calculated numerically. ${ }^{10}$ Finally, the values of $\lambda$ and $E_{r}$ need to be estimated from high-field transport calculations using, for example, Monte Carlo techniques. ${ }^{7}$ The results presented in this paper are compared in Sec. IV to $E_{i}, E_{n}$ and $\lambda$ values obtained from such approaches.

As in the case of $\alpha$ and $\beta$, large discrepancies can be found between the $E_{i}, E_{r}$ and $\lambda$ values reported by different authors (Table I). Any attempt to use a particular set of the three physical parameters from a single reference is unlikely to match the measured ionization coefficient val-

\begin{tabular}{|c|c|c|c|c|c|c|c|}
\hline Material & Type & $E_{i}(\mathrm{eV})$ & $E_{r 0}(\mathrm{meV})$ & $\lambda_{0}(\AA)$ & $E_{r}(\mathrm{meV})^{\mathrm{a}}$ & $\lambda(\dot{A})^{\mathbf{a}}$ & Reference \\
\hline \multirow[t]{4}{*}{$\mathrm{Ge}$} & $\alpha$ & 0.8 & & & 19 & 36 & 3 \\
\hline & $\beta$ & 0.9 & & & 19 & 47 & 3 \\
\hline & $\alpha=\beta$ & & 36 & 105 & & 65 & 8 \\
\hline & $\alpha=\beta$ & & 37 & 105 & & & 13 \\
\hline \multirow[t]{6}{*}{$\mathrm{Si}$} & $\alpha$ & 1.1 & & & 51 & 48 & 3 \\
\hline & $\beta$ & 1.8 & & & 51 & 44 & 3 \\
\hline & $\alpha$ & & 63 & 76 & & 62 & 8 \\
\hline & $\beta$ & & 63 & 47 & & 38 & 8 \\
\hline & $\alpha$ & & 63 & 76 & & & 13 \\
\hline & $\beta$ & & 63 & 55 & & & 13 \\
\hline \multirow[t]{6}{*}{ GaAs } & $\alpha=\beta$ & 1.7 & & & 22 & 33. & 3 \\
\hline & $\alpha=\beta$ & & 36 & 58 & & 35 & 8 \\
\hline & $\alpha=\beta$ & & 35 & 58 & & & 13 \\
\hline & $\beta$ & 1.70 & & & 35 & & 14 \\
\hline & $\alpha=\beta$ & 1.92 & & & 30.0 & 39.3 & 7 \\
\hline & $\alpha=\beta$ & & & & 30.0 & 39.3 & 11 \\
\hline $\mathrm{Al}_{x} \mathrm{Ga}_{1-x} \mathrm{As}$ & $\alpha=\beta$ & & & & $30.0+17.4 x$ & $39.3+15.1 x$ & 11 \\
\hline \multirow[t]{2}{*}{ AlAs } & $\alpha=\beta$ & & & & 47.4 & 54.4 & 11 \\
\hline & $\alpha=\beta$ & 2.80 & $\therefore$ & & 47.4 & 54.4 & 7 \\
\hline \multirow[t]{3}{*}{$\operatorname{InAs}$} & $\alpha$ & $0.42^{b}$ & & & $29^{\mathrm{b}}$ & $170^{b}$ & 18 \\
\hline & $\beta$ & $0.43^{b}$ & & & $29^{b}$ & $400^{\mathrm{b}}$ & 18 \\
\hline & $\alpha=\beta$ & 0.399 & & & 23.8 & 62.9 & 7 \\
\hline \multirow[t]{10}{*}{$\operatorname{In} P$} & $\alpha$ & $1.84^{c}$ & 46 & 41.7 & 32.9 & 29.8 & 9 \\
\hline & $\beta$ & $1.65^{c}$ & 36 & 41.3 & 21.8 & 25.0 & 9 \\
\hline & $\alpha$ & 1.99 & 27 & & 13 & 18.0 & 15 \\
\hline & $\beta$ & 1.65 & 40 & & 26 & 30.1 & 15 \\
\hline & $\alpha$ & & & 37.5 & & & 16 \\
\hline & $\tilde{\beta}$ & & & 42.2 & & & 16 \\
\hline & $\beta$ & 1.55 & & & 43 & & 14 \\
\hline & $\alpha=\beta$ & 1.79 & & & 27.3 & 38.9 & 7 \\
\hline & $\alpha$ & $1.84^{\mathrm{c}}$ & 27.0 & & & & 17 \\
\hline & $\beta$ & $1.65^{\mathrm{C}}$ & 43.0 & & & & 17 \\
\hline \multirow[t]{2}{*}{$\mathrm{In}_{0.53} \mathrm{Ga}_{0.47} \mathrm{As}$} & $\alpha$ & 0.82 & 32.7 & & & & 17 \\
\hline & $\beta$ & 0.83 & 32.7 & & & & 17 \\
\hline \multirow[t]{3}{*}{ GaP } & $\alpha=\beta$ & 2.6 & & & 38 & 31 & 3 \\
\hline & $\alpha=\beta$ & & 50 & 42 & & 32 & 8 \\
\hline & $\alpha=\beta$ & 2.94 & & & 33.0 & 32.5 & 7 \\
\hline \multirow[t]{2}{*}{ InSb } & $\alpha=\beta$ & 0.2 & , & & & & 10 \\
\hline & $\alpha=\beta$ & 0.187 & & & 21.2 & ${ }^{\cdots} 100.2$ & 7 \\
\hline AlP & $\alpha=\beta$ & 3.14 & & & 54.2 & 71.6 & 7 \\
\hline $\mathrm{AlSb}$ & $\alpha=\beta$ & 2.00 & & & 35.4 & 45.3 & 7. \\
\hline $\mathrm{GaSb}$ & $\alpha=\beta$ & 0.848 & & & 24.2 & 48.5 & 7 \\
\hline
\end{tabular}

TABLE I. Measured or calculated impact ionization parameters of various semiconductors in $\langle 100\rangle$ direction.

$300 \mathrm{~K}$ values unless otherwise specified.

${ }^{b} 77 \mathrm{~K}$ values.

${ }^{c}$ Reference 12. 
TABLE II. Temperature-dependence expressions for band-gap energy $E_{g}$ in various semiconductors.

\begin{tabular}{|c|c|c|c|}
\hline Material & $\begin{array}{c}E_{\mathrm{g}} \text { at } 300 \mathrm{~K} \\
(\mathrm{eV})\end{array}$ & $\begin{array}{l}\text { Temperature dependence } \\
\text { of band gap } E_{g}(T)(\mathrm{eV})\end{array}$ & Reference \\
\hline $\mathrm{Ge}$ & $\begin{array}{l}0.663 \\
\text { (indirect) }\end{array}$ & $0.7437-4.774 \times 10^{-4} T^{2} /(T+235)$ & 13 \\
\hline $\mathbf{S i}$ & $\begin{array}{l}1.125 \\
\text { (indirect) }\end{array}$ & $1.170-4.730 \times 10^{-4} T^{2} /(T+636)$ & 13 \\
\hline GaAs & 1.424 & $1.519-5.405 \times 10^{-4} T^{2} /(T+204)$ & 19 \\
\hline $\begin{array}{l}\mathrm{Al}_{x} \mathrm{Ga}_{1-x} \mathrm{As} \\
(x<0.45)\end{array}$ & $1.424+1.247 x$ & $\begin{array}{c}d E_{g}^{\Gamma} / d T=(-3.95-1.15 x) \times 10^{-4} \\
\quad(\text { in } \mathrm{eV} / \mathrm{K} \text { for } T>150 \mathrm{~K})\end{array}$ & 20 \\
\hline AlAs & $\begin{array}{l}2.163 \\
\text { (indirect) }\end{array}$ & $2.239-6.0 \times 10^{-4} T^{2} /(T+408)$ & 19 \\
\hline $\operatorname{InP}$ & 1.351 & $1.421-3.63 \times 10^{-4} T^{2} /(T+162)$ & 19 \\
\hline $\mathrm{In}_{0.53} \mathrm{Ga}_{0,47} \mathrm{As}$ & 0.75 & $\begin{array}{c}d E_{g}^{\Gamma} / d T=-3.71 \times 10^{-4} \\
(\text { in } \mathrm{eV} / \mathrm{K} \text { for } T>150 \mathrm{~K})\end{array}$ & 21 \\
\hline $\mathrm{In}_{0.52} \mathrm{Al}_{0.48} \mathrm{As}$ & 1.48 & $\begin{array}{c}d E_{g}^{\mathrm{r}} / d T=-4.27 \times 10^{-4} \\
\text { (in } \mathrm{eV} / \mathrm{K} \text { for } T>150 \mathrm{~K} \text { ) }\end{array}$ & 20,21 \\
\hline InAs & 0.360 & $0.420-2.50 \times 10^{-4} T^{2} /(T+75)$ & 19 \\
\hline $\mathrm{GaP}$ & $\begin{array}{l}2.261 \\
\text { (indirect) }\end{array}$ & $2.338-5.771 \times 10^{-4} T^{2} /(T+372)$ & 19 \\
\hline InSb & 0.172 & $0.236-2.99 \times 10^{-4} T^{2} /(T+140)$ & 19 \\
\hline AlP & $\begin{array}{l}2.45 \\
\text { (indirect) }\end{array}$ & $2.52-3.18 \times 10^{-4} T^{2} /(T+588)$ & 19 \\
\hline AlSb & $\begin{array}{l}1.58 \\
\text { (indirect) }\end{array}$ & $1.687-4.97 \times 10^{-4} T^{2} /(T+213)$ & 19 \\
\hline GaSb & 0.726 & $0.810-3.78 \times 10^{-4} T^{2} /(T+94)$ & 19 \\
\hline
\end{tabular}

ues. In most comparisons, it has therefore been common to use values of one or two of these three fundamental parameters and obtain the remaining one(s) by fitting. Another possibility is to make use of one or two of these parameters as obtained from individual measurements and theoretically predict the values of the ionization coefficients. However, in neither of these approaches do the results match the measured data. The method proposed in this paper makes direct use of the theoretical model to fit measured ionization coefficients using all $E_{i}, E_{r}$ and $\lambda$ as adjustable physical parameters. Since the model contains approximations and the experimental results are limited in scope and in precision, judgement is required in selecting the most suitable values of $E_{i}, E_{n}$ and $\lambda$; otherwise, the values obtained may lack physical significance. The objective of this paper is to obtain values of $E_{i}, E_{n}$ and $\lambda$ consistent with their meaning in the model, while matching experimental data and providing a realistic estimation of characteristics outside the range of experimental data. Details of the adopted approach are presented in Section III.

Table I summarizes the published data of $E_{i}, E_{n 0}, E_{n}$ $\lambda_{0}, \lambda$ of various semiconductors as reported in the literature based on the model of Baraff or that of Okuto and Crowell. These results demonstrate the existence of discrepancies among the data. The temperature dependence relationships of $E_{i}$ can be assumed to be the same as those for $E_{g}$ as shown in Table II. A simple analytic estimation of $E_{i}$ was made based on the material parameters reported in Refs. 20-23 and is presented in Sec. IV for comparison.

\section{PRACTICAL CONSIDERATIONS IN FITTING MEASURED IMPACT IONIZATION COEFFICIENTS}

The electric-field dependence of the impact ionization coefficients ( $\alpha$ and $\beta$ ) has been found to obey the simple empirical expression of Eq. (1) over the limited range of electric fields considered in the measurements. Extrapolations of such empirical expressions can often result in incorrect tendencies at high or low fields. Figure 1 demonstrates the use of these empirical formulas for fitting the measured impact ionization coefficients of electrons in GaAs (Ref. 24) and $\mathrm{Al}_{x} \mathrm{Ga}_{1-x} \mathrm{As}(x=0.1-0.4){ }^{25}$ The measured data are represented by scattered dots whereas the extrapolated characteristics are represented by lines. The deficiencies of using the simple empirical formula of Eq. (1) for extrapolation are obvious. Since AlGaAs is a wide-band-gap material, its ionization coefficients are expected to be lower than those in GaAs. As can be seen in Fig. 1, this tendency is experimentally verified in the electric-field range of $2.5-4 \times 10^{5} \mathrm{~V} / \mathrm{cm}$. At higher or lower electric fields, the empirical formulas tend to give incorrect tendencies which are indicated by the crossover of the extrapolated curves. This limits the usefulness of these empirical formulas in calculations of avalanche breakdown characteristics of electronic and optical devices. Furthermore, the fitting parameters $A, B$, and $m$ do not have any physical significance. [The advantages of the adopted approach in resolving the crossover of the extrapolated characteristics will be seen below in Sec. IV where the results of 


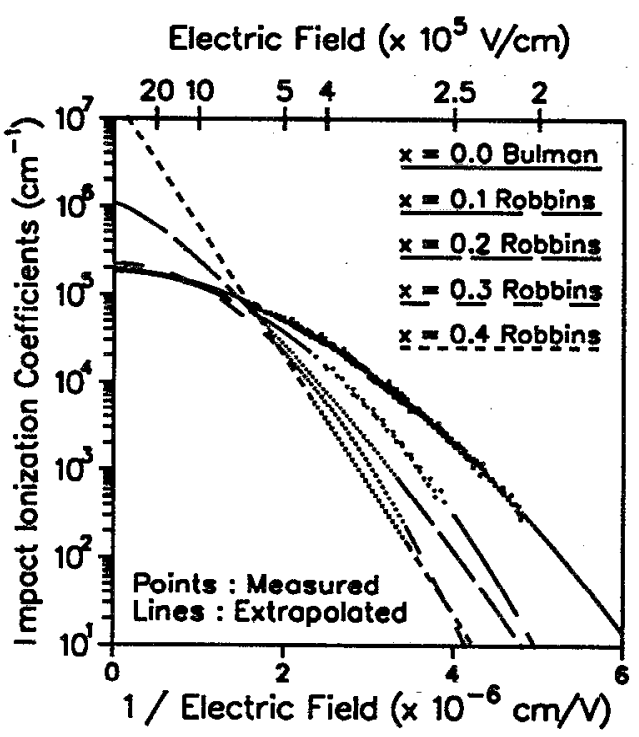

FIG. 1. Extrapolation of measured electron impact ionization coefficients in $\mathrm{GaAs}$ and $\mathrm{Al}_{x} \mathrm{Ga}_{1-x} \mathrm{As}(x=0.1-0.4)$ using the simple empirical formula of Eq. (1). The measured data are represented by scattered dots and the extrapolated curves are represented by lines.

Fig. 1 are compared to Fig. 4(a) for GaAs and $\mathrm{Al}_{x} \mathrm{Ga}_{1-x}$ As $(x=0.1-0.4)$.]

The expected tendencies of impact ionization coefficients as a function of electric field are shown in Fig. 2 for various III-V materials. These results are based on Okuto and Crowell's physical model ${ }^{3}$ in an attempt to fit (and also correct the high-field limit of) Baraff's numerical results of impact ionization coefficients in semiconductors for all electric-field values ${ }^{4}$ (see Sec. II). The ionization coefficient parameters of the III-V materials are obtained by Hauser in his Monte Carlo high-field transport studies. ${ }^{7}$ Equal electron and hole ionization rates were assumed for this purpose. As shown in the figure, the ionization rates tend to infinity as the electric field becomes infinitely large. This characteristic is accounted for in Okuto and Crowell's model in their attempt to correct Baraff's results at high fields. Furthermore, there are no crossover points in the electric-field dependence of the ionization coefficients, as one would expect by properly choosing or calculating $E_{i}$, $E_{n}$ and $\lambda$. The approach adopted in this paper and described next is based on Okuto and Crowell's model to fit published data of measured impact ionization coefficients at $300 \mathrm{~K}$ in some common III-V semiconductors including GaAs, $\mathrm{Al}_{x} \mathrm{Ga}_{1-x}$ As $(x=0.1-0.4), \operatorname{InP}, \mathrm{In}_{0.53} \mathrm{Ga}_{0.47} \mathrm{As}$, and $\mathrm{In}_{0.52} \mathrm{Al}_{0.48} \mathrm{As}$.

Since the discrepancies between the experimental results by different authors can often be much larger than their ranges of experimental uncertainties quoted by them, ${ }^{1}$ the various data available in the literature have to be carefully examined before they can be applied to any analysis of device characteristics. The following four criteria have therefore been employed here in selecting appropriate measured ionization coefficients suitable for fitting: (a) curvature of the plots of measured coefficients with electric field;

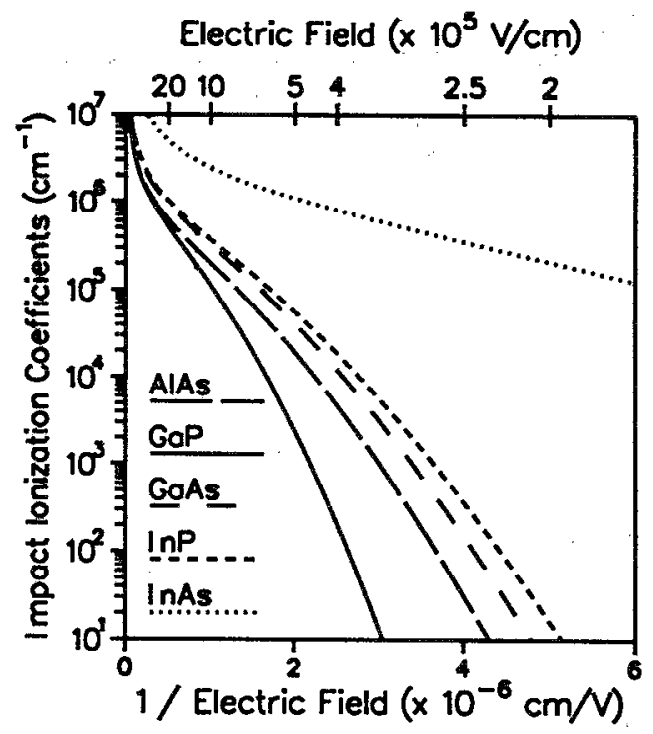

FIG. 2. Theoretical impact ionization coefficients in III-V materials calculated using the model of Okuto and Crowell (Ref. 3) and ionization parameter data from Ref. 7.

(b) agreement of the measured data with the theories referred to in Refs. 3, 8, 10,12, and 14; (c) comparison of $\alpha, \beta$ versus electric-field features in various materials; and (d) range of electric fields covered in the measurements. After selection of the most reliable measured data, Okuto and Crowell's theoretical model [Eq. (2)] was used for fitting them. A least-squares fitting was used and the fitting parameters were $\ln (\alpha)$ and $\ln (\beta)$. For the three fundamental parameters $E_{i}, E_{r}$ and $\lambda$ to maintain their physical significance, it was necessary to perform the optimization by restricting the range of their possible values. An appropriate choice of this range is necessary to ensure the effectiveness of the model in predicting meaningful impact ionization coefficients at very. large and small electric fields normally not considered in the experiments. The scatter of the measured data reported by different authors and the discrcpancies observed between theoretical and experimental results are likely to be due to systematic errors in the experiments. ${ }^{1}$ In this work it is not, therefore, attempted to make a perfect fit to the "most reliable" sets of experimental data (which can still be subjected to systematic errors) by sacrificing the physical meaning of $E_{i}, E_{n}$ and $\lambda$. Instead, fitting of the data is compromised in order to obtain a meaningful physical model capable of extrapolating impact ionization coefficients over a wide range of electric fields and temperatures. The results can be useful in avalanche breakdown calculations of electronic and optical devices.

\section{FITTED AND EXTRAPOLATED RESULTS IN GaAs, $A I_{x} \mathrm{Ga}_{1-x} \mathrm{As}$, InP, $\operatorname{In}_{0.53} \mathrm{Ga}_{0.47}$ As AND In $_{0.52} \mathrm{Al}_{0.48} \mathrm{As}$}

\section{A. GaAs}

GaAs is by far the most extensively studied material among all III-V semiconductors. Numerous reports have 


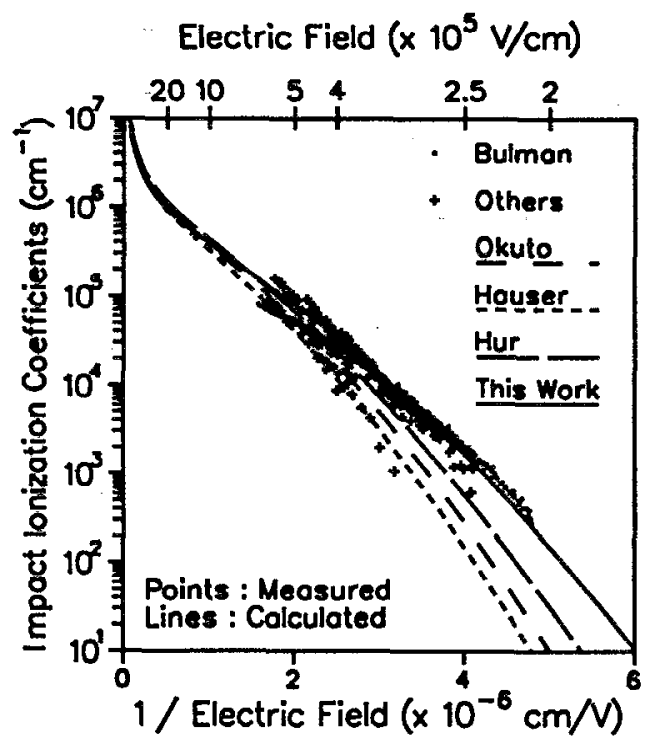

(a)

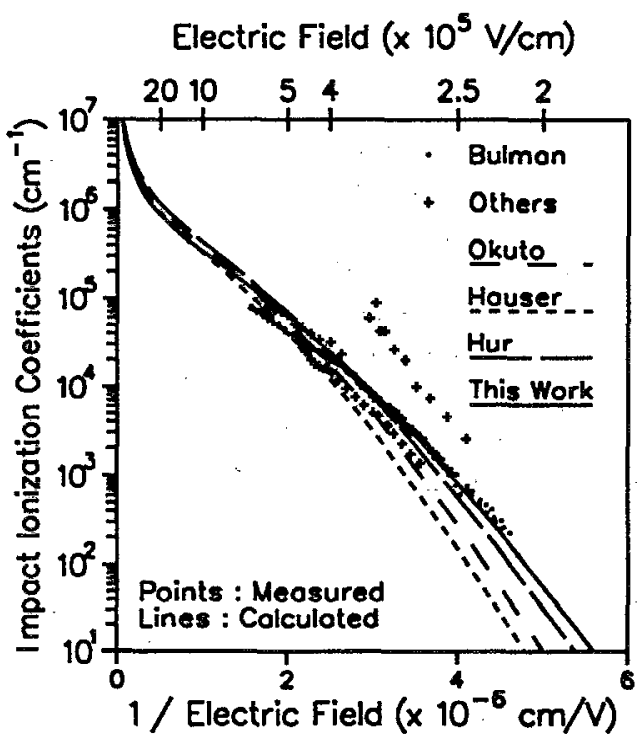

(b)

FIG. 3. Comparison between the impact ionization coefficients in GaAs as estimated in this work (solid line) and as obtained by measurements (Ref. 24: scattered dots). The experimental results (Refs. 27-32: scattered crosses) and the theoretical results of various groups (Refs. 3, 7, and 11: broken lines) are included for comparison; (a) refers to electron and (b) to hole ionization coefficients.

been published over the past few decades on measured impact ionization coefficients in GaAs. The large discrepancies found between the results of different groups (see for example the review in Ref. 26) prompted Bulman and co-workers to undertake a systematic and detailed study of the ionization rates in a large number of GaAs wafers and diodes. $^{24}$

Figures 3(a) and 3(b) shows the fitted (and extrapolated) results (solid line) of the measured coefficients $\alpha$ and $\beta$ for the case of GaAs (Bulman and co-workers' data: scattered dots); Eq. (2) was used for this purpose. The experimental results (scattered crosses) of Ito et al., ${ }^{27}$ Pearsall, Nahory, and Chelikowsky, ${ }^{28}$ Stillman et al., ${ }^{29}$
David et al. ${ }^{30}$ Law and Lee, ${ }^{31}$ and Ando and Kanbe, ${ }^{32}$ and the theoretical results of Okuto and Crowell, ${ }^{3} \mathrm{Hauser},{ }^{7}$ and Hur and co-workers ${ }^{11}$ are also included for comparison.

As can be observed from the figure, all theoretical data and the results of this work show slightly different rates of change with electric field compared to those of Bulman's measured data. Moreover, the experimental errors shown in each set of data are much less than the discrepancies between different sets of measured data. We therefore do not attempt to obtain a perfect fitting to any particular set of measured data (or within the quoted error bars of that particular set of measured data), but rather to extract the three physical parameters $\left(E_{i}, E_{n}\right.$ and $\left.\lambda\right)$ for extrapolation of ionization coefficients. As shown in Figs. 3(a) and 3(b), our results are well within the "error bars" represented by the results of different groups in the range of electric fields covered in the experiments. An attempt to perfectly fit Bulman and co-workers' measured data using the theoretical model results in values of $E_{i}, E_{r}$ and $\lambda$ that deviate excessively from their physically expected values. $E_{i}$ becomes, for example, too large (several hundred eV). This was therefore avoided in the adopted approach. Furthermore, as will be seen below (Fig. 4), the measured ionization coefficients in GaAs show a stronger electric-field dependence than in $\mathrm{Al}_{x} \mathrm{Ga}_{1-x}$ As. The measured $\alpha$ and $\beta$ values in GaAs level off more rapidly than in $\mathrm{Al}_{x} \mathrm{Ga}_{1-x} \mathrm{As}$, and tend to cross over the $\mathrm{Al}_{x} \mathrm{Ga}_{1-x}$ As data at high fields. Our adopted approach corrected this behavior and gives reasonably good estimates of characteristics at high fields. In traditional approaches where the sole objective is perfect fitting to measured data, there is a significant deviation between the theoretical (or fitting) results and measured data at high fields.

The ionization parameters $E_{i}, E_{n}$ and $\lambda$ evaluated in this work are presented in Table III for various materials. A simple estimation of $E_{i}^{e}$ and $E_{i}^{h}$ is also included in the table for comparison using the analytic expressions given in the Appendix. This estimation is based on both equal and unequal electron and hole effective-mass assumptions with the material parameters being taken and interpolated from Refs. $20-23$. In the former case, $E_{i}^{e}=1.5 E_{g}$, while in the latter case, $E_{i}^{e}$ was found to be about the same as $E_{i}^{h}$ and approximately 1.1 times the band-gap energy $E_{g}$ $\left(E_{i}^{e} \approx E_{i}^{h}=1.1 E_{g}\right.$ ). As can be observed from Table III, the fitted results of $E_{i}$ are close to the values estimated from the equal-effective-mass assumption. This agreement is in general observed in other materials as well, including $\mathrm{Al}_{x} \mathrm{Ga}_{1-x} \mathrm{As}$, InP, $\mathrm{In}_{0.53} \mathrm{Ga}_{0.47} \mathrm{As}$, and $\mathrm{In}_{0.52} \mathrm{Al}_{0.48} \mathrm{As}$ (see results of Table III).

\section{B. $\mathrm{Al}_{x} \mathrm{Ga}_{1-x} \mathrm{As}$}

In contrast to GaAs, very few reports have been made on both theoretically calculated and experimentally measured impact ionization coefficients in $\mathrm{Al}_{x} \mathrm{Ga}_{1-x} \mathrm{As}$. The experimental work by Robbins and co-workers ${ }^{25}$ is probably the most extensive treatment of this material to date for composition $x$ ranging from 0.1 to 0.4 . A recent work by Hur and co-workers theoretically calculates the ionization 
TABLE III. Impact ionization parameters of various III-V semiconductors in $(100\rangle$ direction at $300 \mathrm{~K}$ as estimated analytically and as obtained by fitting in this work.

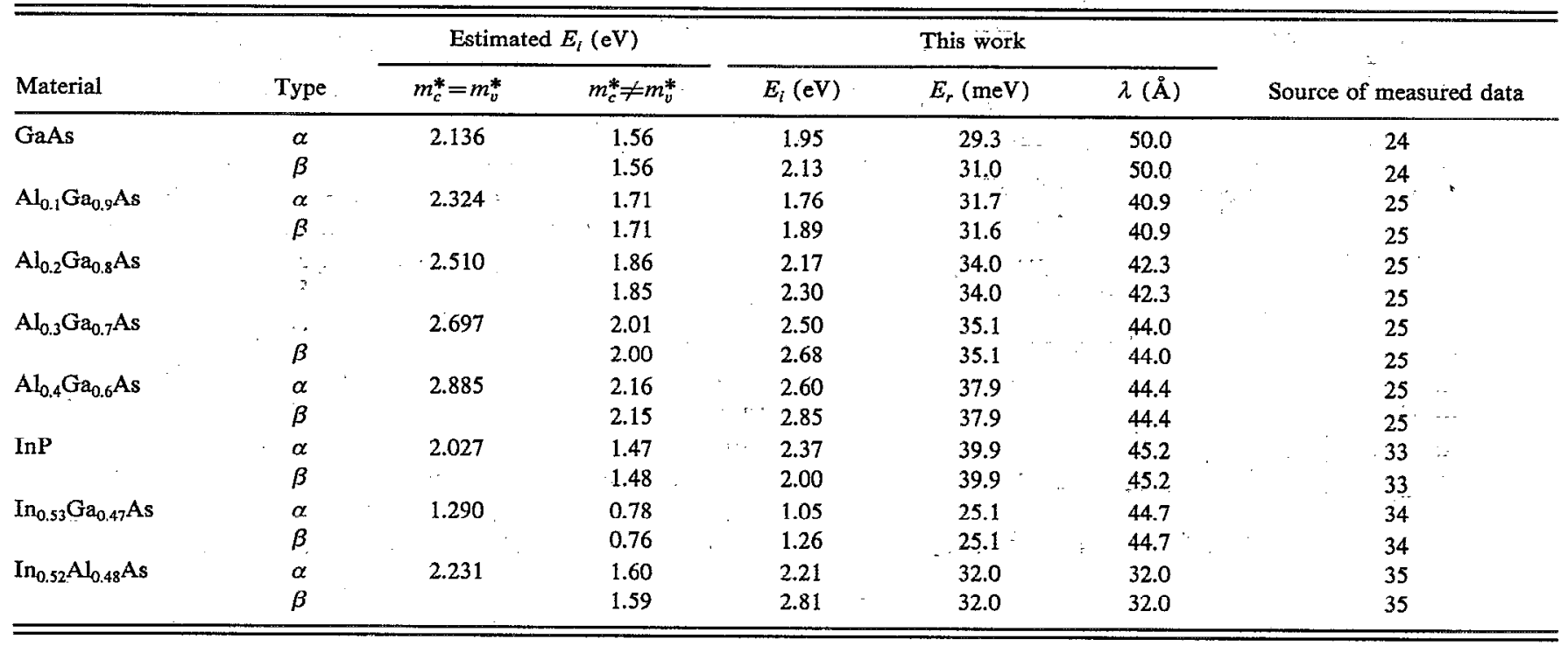

rates (assuming $\alpha=\beta$ ) in this material for all $x$ based on Okuto and Crowell's model and Eqs. (2)-(4) and (A1)(A2). ${ }^{11}$ The variation in $\alpha, \beta$ with $x$ in their work is not, however, adequately compared to measured data.

The fitted and extrapolated results for $\mathrm{Al}_{x} \mathrm{Ga}_{1-x} \mathrm{As}(x$ $=0.0-0.4$ ) are shown in Fig. 4, as obtained by the approach used in this paper. In contrast to Fig. 1, these results show ionization coefficient versus field trends that do not cross over for different $\mathrm{Al}$ compositions $\boldsymbol{x}$ except at very large electric fields $\left(>11 \times 10^{5} \mathrm{~V} / \mathrm{cm}\right)$ and only for the $x=0.0$ and $x=0.1$ cases. As already mentioned in Sec. III, the rate of change with electric field of the measured ionization coefficients is faster in GaAs than in $\mathrm{Al}_{x} \mathrm{Ga}_{1-x}$ As. An excellent agreement is observed between the fitted results and the measured data for $\mathrm{Al}_{x} \mathrm{Ga}_{1-x} \mathrm{As}$. The extrapolated $\alpha$ and $\beta$ are obtained by Okuto and Crowell's physical model based on the three extracted physical parameters, and the model itself has been verified by Baraff's numerical analysis over the whole range of electric fields. Without the guidance of a physical model and accurate physical parameters, extrapolation of impact ionization coefficients would be a difficult task. Since the range of electric fields considered is beyond experimental limits, the validity of the extrapolated values can only be verified through numerical analysis. Unlike the extrapolated curves shown in Fig. 1, the trends of the $\alpha, \beta$ versus electric-field characteristics in $\mathrm{Al}_{x} \mathrm{Ga}_{1-x}$ As are maintained outside the regions of measured data, i.e., at very high and low electric fields. This demonstrates the appropriateness of the adopted technique for fitting measured data and extrapolating beyond them, while conserving the features predicted by physical modeling.

A further verification of the appropriatencss of the technique has been obtained by introducing the empirically determined expressions for $\alpha$ and $\beta$ in a simulator of HEMT (high electron mobility transistor) breakdown. ${ }^{2}$ When these expressions are extrapolated and used outside the range of measured data, the breakdown characteristics of the device show trends that are not seen in practice, i.e., an increased breakdown voltage for lower $\mathrm{Al}$ composition. The correct breakdown trends can only be obtained if the $\alpha$ and $\beta$ versus electric-field curves do not cross each other, as expected by the physical model of Okuto and Crowell ${ }^{3}$ and proposed in this work.

The results for GaAs and $\mathrm{Al}_{x} \mathrm{Ga}_{1-x}$ As obtaincd in this work and summarized in Table III show $E_{i}$ and $\lambda$ values for GaAs that are slightly higher than what one would expect for these parameters from the AlGaAs trends when $x$ approaches zero. This is probably due to differences between the measurement procedures reported by Refs. 24 and 25 for $\mathrm{AlGaAs}$. The equal-effective-mass assumption seems to agree better with the optimized $E_{i}$ 's. Finally $E_{r}$ and $\lambda$ are comparable to the data reported by other research groups and shown in Table I.

\section{InP}

Table III also includes the fitted ionization parameters $E_{b}, E_{n}$ and $\lambda$ for InP, together with a simple estimation of $E_{i}^{e}$ and $E_{i}^{h}$ based on both equal and unequal electron and hole effective-mass assumptions. As was observed for other materials, the $E_{i}^{e}$ values estimated based on the latter assumption were found to be about the same as those of $E_{i}^{h}$, and approximately equal to 1.1 times the band-gap energy $E_{g}$. The larger measured hole ionization coefficients in InP results in larger $E_{i}$ values for holes than for electrons. The optimized $E_{i}$ 's are, however, close to the estimated values based on the equal-effective-mass assumption. The fitted results for $E_{i}, E_{n}$ and $\lambda$ are comparable to the data reported by the other research groups, as given in Table I.

\section{D. $\operatorname{In}_{0.53} \mathrm{Ga}_{0.47} \mathrm{As}$}

$\mathrm{In}_{0.53} \mathrm{Ga}_{0.47} \mathrm{As}$ is an important III-V semiconductor for device applications because of its superior electrical prop- 


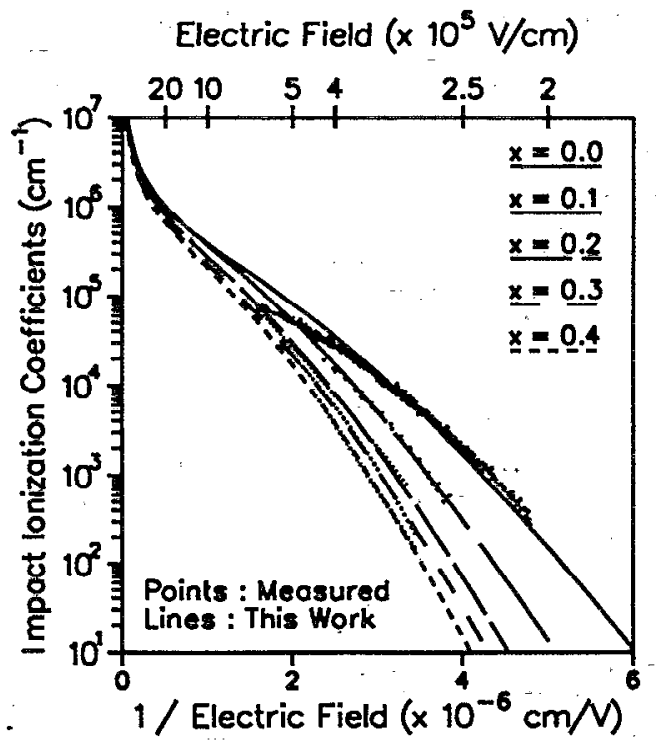

(a)

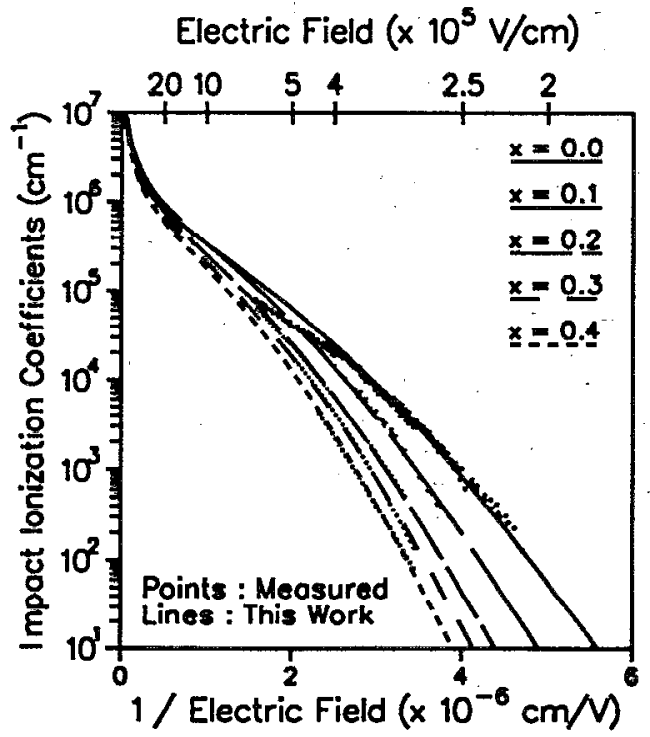

(b)

FIG. 4. Impact ionization coefficients in $\mathrm{Al}_{x} \mathrm{Ga}_{1-x} \mathrm{As}$ as estimated in this work (lines) and as obtained by experiments (Ref. 25: scattered dots) for $x=0.1,0.2,0.3$, and 0.4 . The results of this work for $x=0$ are also included for comparison (see also Fig. 3); (a) refers to electron and (b) to hole ionization coefficients.

erties over GaAs. Very few reports have, however, been made on the measurement of ionization coefficients in this material. The work by Pearsall ${ }^{34}$ and by Osaka and coworkers ${ }^{17}$ is most commonly used for devices containing $\mathrm{In}_{0.53} \mathrm{Ga}_{0.47}$ As. Almost an order of magnitude difference in the measured ionization rates are reported by Refs. 17 and 34, which makes the selection of the experimental data and our purposes of fitting very difficult. The optimized $E_{i}, E_{r}$ and $\lambda$ for Pearsall's data are shown in Table III, as obtained by applying the approach presented in this paper.

\section{E. $\ln _{0.52} \mathbf{A l}_{0.48} \mathrm{As}$}

$\operatorname{In}_{0.52} \mathrm{Al}_{0.48} \mathrm{As}$ is another important wide-band-gap material because of its lattice matching to both InP and
$\mathrm{In}_{0.53} \mathrm{Ga}_{0.47} \mathrm{As}$ and the possibility of forming various heterojunctions with useful characteristics. Unfortunately, as in the case of $\mathrm{In}_{0.53} \mathrm{Ga}_{0.47} \mathrm{As}$, very few reports exist for the measured impact ionization coefficients in this material. The works by Capasso et $a .^{36}$ and by Watanabe et $a .^{35}$ are the prime sources for measured data with this material. A linear interpolation was used in this case for the analytic estimation of $E_{i}$ 's due to the lack of complete information on the material properties. The optimized $E_{i}^{e}$ and $E_{i}^{h}$ values for the data of Watanabe et al. compare reasonably well with estimated values as shown in Table III.

\section{CONCLUSIONS}

The physical model and theoretical expressions derived by Okuto and Crowell have been used to fit impact ionization experimental data at $300 \mathrm{~K}$ for. $\mathrm{GaAs}, \mathrm{Al}_{x} \mathrm{Ga}_{1-x} \mathrm{As}$ $(x=0.1-0.4)$, InP, $\operatorname{In}_{0.53} \mathrm{Ga}_{0.47} \mathrm{As}$, and $\mathrm{In}_{0.52} \mathrm{Al}_{0.48} \mathrm{As}$. It was found that the expressions derived by the proposed approach are consistent not only over the range of measured datä but are also plausible at very large or small electric fields not covered by the measurements. This approach yielded values with physical significance for parameters such as the ionization threshold energy $E_{i}$, average energy loss per phonon scattered $E_{r}$ and mean free path for optical phonons $\lambda$. The optimized ionization coefficient parameters, coupled with the temperature-dependence capability of Okuto and Crowell's model, can be used to predict impact ionization coefficients over a wide range of electric fields and temperatures. They are consequently very useful in simulating device performance, such as the avalanche breakdown characteristics of electronic devices operating at various temperatures.

\section{ACKNOWLEDGMENT}

The support of this work by the U.S. Army Research Office under Contract No. DAAL03-87-K-0007 is greatly acknowledged.

\section{APPENDIX: CALCULATION OF IMPACT IONIZATION THRESHOLD ENERGY $E_{i}$}

The impact ionization threshold energy $E_{i}$ can be calculated using the simple analytic expressions summarized below. The values of $E_{i}$ obtained from these expressions can be compared with those obtained using the approach presented in this paper (see, for example, the comparison presented in Table III).

\section{Electron-initiated Ionization}

(i) Direct band gap: ${ }^{10}$

$$
E_{i}^{e}=E_{g}\left(1+\frac{m_{c}^{*}}{m_{c}^{*}+m_{v}^{*}}\right) .
$$

For equal electron and hole effective masses, $E_{i}$ becomes $\frac{3}{2}$ of the band gap $E_{g}$, which has frequently been used to estimate ionization threshold energies.

(ii) Indirect band gap: $:^{11}$ 


$$
\begin{aligned}
E_{i}^{*}= & E_{g}\left(1+\frac{m_{c}^{*}}{m_{c}^{*}+m_{v}^{*}}\right)+E_{m}\left\{\frac{m_{c}^{*}\left(3 m_{c}^{*}+m_{v}^{*}\right)}{\left(m_{c}^{*}+m_{v}^{*}\right)^{2}}-\frac{2 m_{c}^{*}}{m_{c}^{*}+m_{v}^{*}}\right. \\
& \left.\times\left[\left(1+\frac{m_{c}^{*}}{m_{c}^{*}+m_{v}^{*}}\right)\left(\frac{m_{c}^{*}}{m_{c}^{*}+m_{v}^{*}}+\frac{E_{g}}{E_{m}}\right)\right]^{1 / 2}\right\} \cdot
\end{aligned}
$$

\section{Hole-initiated ionization (Ref. 12)}

$$
E_{i}^{h}=E_{g}\left(1+\frac{m_{\mathrm{so}}^{*}\left(1-\Delta / E_{g}\right)}{2 m_{v}^{*}-m_{\mathrm{s}-\mathrm{o}}^{*}+m_{c}^{*}}\right),
$$

where $E_{g}$ is the direct (or indirect) energy gap, $E_{m}$ $=\left(\hbar^{2} / 2 m_{c}^{*}\right)(2 \pi / a)^{2}, \Delta$ is the spin-orbit splitting of the valence band, $m_{c}^{*}$ is the direct (or indirect) band-gap conduction-band electron effective mass, $m_{v}^{*}$ is the valenceband hole effective mass, $m_{s-0}^{*}$ is the spin-orbit split-off band hole effective mass, and $a$ is the lattice constant.

${ }^{1}$ A. S. Kyuregyan and S. N. Yurkov, Sov. Phys. Semicond. 23, 1126 (1989).

${ }^{2}$ H.-F. Chau, D. Pavlidis, and K. Tomizawa, IEEE Trans. Electron Devices ED-38, 213 (1991).

${ }^{3}$ Y. Okuto and C. R. Crowell, Phys. Rev. B 6, 3076 (1972).

${ }^{4}$ G. A. Baraff, Phys. Rev. 128, 2507 (1962).

${ }^{5}$ W. Shockley, Solid-State Electron. 2, 35 (1961).

${ }^{6}$ P. A. Wolff, Phys. Rev. 95, 1415 (1954).

${ }^{7}$ J. R. Hauser, Appl. Phys. Lett. 33, 351 (1978).

${ }^{8}$ C. R. Crowell and S. M. Sze, Appl. Phys. Lett. 9, 242 (1966).

${ }^{9}$ K. Taguchi, T. Torikai, Y. Sugimoto, K. Makita, and H. Ishihara, J. Appl. Phys. 59, 476 (1986).

${ }^{10}$ C. L. Anderson and C. R. Crowell, Phys. Rev. B 5, 2267 (1972).

${ }^{11}$ J. H. Hur, C. W. Myles, and M. A. Gundersen, J. Appl. Phys. 67, 6917 (1990).

${ }^{12}$ T. P. Pearsall, Appl. Phys. Lett. 35, 168 (1979).

${ }^{13}$ S. M. Sze, Physics of Semiconductor Devices (Wiley, New York, 1981).

${ }^{14} \mathrm{~K}$. Brennan and K. Hess, Phys. Rev. B 29, 5581 (1984).
${ }^{15}$ C.-W. Kao and C. R. Crowell, Solid-State Electron. 23, 881 (1980).

${ }^{16}$ Y. Takanashi and Y. Horikoshi, Jpn. J. Appl. Phys. 20, 1907. (1981).

${ }^{17}$ F. Osaka, T. Mikawa, and T. Kaneda, IEEE J. Quantum Electron. QE-21, 1326 (1985).

${ }^{18}$ M. P. Mikhailova, N. N. Smirnova, and S. V. Slobodchikov, Sov. Phys. Semicond. 10, 509 (1976).

${ }^{19}$ H. C. Casey and M. B. Panish, Heterostructure Lasers: Part AFundamental Principles; Part B-Materials and Operating Characteristics (Academic, New York, 1978).

${ }^{20}$ S. Adachi, J. Appl. Phys. 58, R1 (1985).

${ }^{21}$ S. Adachi, J. Appl. Phys. 53, 8775 (1982).

${ }^{22}$ D. Olego, T. Y. Chang, E. Silberg, E. A. Caridi, and A. Pinczuk, Appl. Phys. Lett. 41, 476 (1982).

${ }^{23}$ M. A. Littlejohn, T. H. Glisson, and J. R. Hauser, in GaInAsP Alloy Semiconductors, edited by T. P. Pearsall (Wiley, New York, 1982).

${ }^{24}$ G. E. Bulman, V. M. Robbins, and G. E. Stillman, IEEE Trans. Electron Devices ED-32, 2454 (1985).

${ }^{25}$ V. M. Robbins, S. C. Smith, and G. E. Stillman, Appl. Phys. Lett. 52, 296 (1988).

${ }^{26}$ G. E. Stillman, V. M. Robbins, and N. Tabatabaie, IEEE Trans. Electron Devices ED-31, 1643 (1984).

${ }^{27}$ M. Ito, S. Kagawa, T. Kaneda, and T. Yamaoka, J. Appl. Phys. 49, 4607 (1978).

${ }^{28}$ T. P. Pearsall, R. E. Nahory, and J. R. Chelikowsky, Phys. Rev. Lett. 39, 295 (1977).

${ }^{29}$ G. E. Stillman, C. M. Wolfe, J. A. Rossi, and A. G. Foyt, Appl. Phys. Lett. 24, 471 (1974).

${ }^{30}$ J. P. R. David, J. S. Marsland, H. Y. Hall, G. Hill, N. J. Mason, M. A. Pate, J. S. Roberts, P. N. Robson, J. E. Sitch, and R. C. Woods, Inst. Phys. Conf. Ser. 74, 247 (1984).

${ }^{31}$ H. D. Law and C. A. Lee, Solid-State Electron. 21, 331 (1978).

${ }^{32} \mathrm{H}$. Ando and H. Kanbe, Solid-State Electron. 24, 629 (1981).

${ }^{33}$ L. W. Cook, G. E. Bulman, and G. E. Stillman, Appl. Phys. Lett. 40, 589 (1982).

${ }^{34}$ T. P. Pearsall, Appl. Phys. Lett. 36, 218 (1980).

${ }^{35}$ I. Watanabe, T. Torikai, K. Marita, K. Fukushima, and T. Uji, IEEE Electron Device Lett. EDL-11, 437 (1990).

${ }^{36}$ F. Capasso, K. Mohammed, K. Alavi, A. Y. Cho, and P. W. Foy, Appl. Phys. Lett. 45, 968 (1984). 